Eye tests

\section{Use of Lea symbols in young children}

\section{X Repka}

\section{Clinicians must continue to refine as well as develop new visual acuity tests for preschool children}

A ccurate measurement of visual acuity is fundamental to ophthalmological care for all ages. This measure is used for diagnosis, for documentation of clinical outcomes, for qualification for special education or disability programmes, and for permission to drive a motor vehicle. More than 20 years ago the importance of ensuring that such a measure be scientifically rigorous was recognised with the development of the Bailey-Lovie visual acuity test ${ }^{1}$ and the Early Treatment of Diabetic Retinopathy Study (ETDRS) test derived from it. ${ }^{2}$ The ETDRS test incorporated important design elements including letter selection, proportional optotype spacing, standard illumination, methods of administration, and scoring. Despite the quality of this test for school age children and adult patients, it is too difficult for preschool children.

Clinicians testing visual acuity of preschool children have not yet found a single best test. An accurate, reproducible, and highly testable measure is needed by the paediatric eye care community for screening and office testing. Practitioners have utilised an assortment of test chart configurations with letter, number, and picture optotypes. Letter optotypes have generally been too difficult for preschool children, while traditional picture optotypes, like the Allen pictures, though much more testable, substantially reduce the sensitivity of the test for the detection of amblyopia. ${ }^{34}$ This is a critical drawback since amblyopia is the most common reason to test visual acuity in this age group. In addition, the practitioner has individualised most testing with plenty of room for the art of administration, including the judicious use of second chances.

An ideal test of visual acuity in early childhood should include as many of the design features of the ETDRS as possible. Using the same test optotypes and presentation strategy throughout childhood would eliminate the measurement variability that is introduced by using a different test at each stage of therapy as the child ages. The ideal test should be constructed in a regular logMAR progression to facilitate detection of change at all levels of acuity. The test should utilise single optotypes, which increase testability in children. ${ }^{5}$ However, single optotypes are not as sensitive to amblyopia as linear optotypes, owing to the absence of contour interaction with the single optotypes. Crowding bars or even a surround box should be placed 0.5 letter width away from the test optotype to improve the test sensitivity. ${ }^{67}$ The test should incorporate letter optotypes, which are more sensitive to amblyopia than the pictures often used (for example, Allen), unless a picture test that is as sensitive to amblyopia can be devised. The single surrounded HOTV is a good example. ${ }^{8}$ The surround bars combined with single letters produce a result that closely approximates line letter acuity. The Glasgow acuity cards are a test which uses letter optotypes (X V O H U $\mathrm{Y})$ presented as groupings of four in a surround box ensuring contour interaction. $^{9-11}$ Random presentation of optotypes is preferred to avoid memorisation. Commercially available computerised display systems would seem to be ideally suited for this task compared to printed charts.

\section{An accurate, reproducible, and highly testable measure is needed by the paediatric eye care community for screening}

A specific and reproducible testing protocol should be part of the test as it is with the ETDRS. The protocol will probably use a modified staircase, rather than asking the preschool child to identify every optotype in the test, to reach threshold more quickly and improve overall testability. ${ }^{10}$ One such strategy was developed and implemented for the Amblyopia Treatment Study (ATS). ${ }^{8}$ This measurement protocol used single surrounded HOTV optotypes. It had high testability between age 4 and 7 years $(92 \%)$, though it could be performed by only about $50 \%$ of children between 2 and 4 years of age. It is this reduced testability of even the simplest crowded letter optotypes in young children that has kept clinicians searching for a picture based optotype test for use in younger preschool children. ${ }^{12}$
In this issue of the BJO (p 513) Becker and his colleagues extend their work in preschool visual acuity testing with the Lea symbols. Dr Lea Hyvärinen designed a set of tests based on picture optotypes for use in children. ${ }^{13}$ They were designed to have contours like the Landolt C. These tests make use of common pictures felt to improve testability among young children and eliminate cultural biases. ${ }^{13-15}$ They have become very popular among clinicians. It is hoped that these optotypes will exhibit sensitivity for amblyopia equivalent to letter optotypes.

Becker and colleagues report on the results of two projects. In the first, the single Lea symbols distance test was used in a paediatric practice on unselected children from 21 to 93 months of age (median 47 months). Overall, the testability was a disappointing $54 \%$. The testability after age 4 years was quite good $(93 \%)$, but these older children are in the age range when letter based tests can be successfully administered. ${ }^{81011}$ More troublesome was the poor testability between 21 and 48 months, when only $31 \%$ of children completed the testing. This is just the age when a sensitive picture based optotype test would be revolutionary. Though the performance of the Lea tests in this setting was disappointing, other authors have found better testability with Lea symbols, both in isolated and line versions, in children between 3 and 4 years of age. ${ }^{14} 16$

The second study in this report compared the single Lea symbols to the crowded Lea symbols as well as to single and crowded Landolt $\mathrm{C}$ tests. The crowded Landolt $\mathrm{C}$ test was designed with fixed rather than proportional interoptotype spacing, which may impact the sensitivity of the test. The study was performed in an ophthalmology clinic setting and included normal children from 23 to 70 months of age. Single Lea picture testability was better in this clinic setting, $77 \%$, though it was only $56 \%$ before 48 months of age. The crowded Lea symbols were more difficult to successfully complete, with only $57 \%$ of studied children able to complete the test. As might be predicted both versions of the Lea symbols test had higher testability than the Landolt C tests. However, the surrounded Lea tests overestimated acuity by 1.9 lines compared to the crowded Landolt $\mathrm{C}$ in normal eyes.

Reproducibility was not directly assessed. However, it is possible to infer such a rate from the intereye difference of this visually normal population by assuming the acuities of each eye are equal. The measurements for the two eyes were within one $\log$ MAR line for the single Lea symbols, either better or worse, in only $84 \%$ of the children screened in paediatric offices. This means that when a one line change is observed, $16 \%$ of the time it does not represent 
actual change. This imprecision was noted for all ages tested. Better test reproducibility was noted during ophthalmological office testing, with about $90 \%$ of patients within one line on retest for both the single and surrounded Lea symbols. Similar rates of $95 \%$ and $93 \%$ have been reported for the Glasgow acuity cards $^{10}$ and the single surrounded HOTV (ATS protocol) respectively. ${ }^{8}$

These studies did not address the crucial issue of the sensitivity of the Lea symbols in single or surrounded format for the detection of amblyopia. The authors have previously reported on the sensitivity of Lea symbols in older strabismic amblyopes. ${ }^{17}$ In that study the Lea test with crowded symbols overestimated visual acuity by about two $\log$ MAR lines compared to a crowded Landolt C.

It would seem logical for future testing that the surrounded versions of the Lea test should be preferred based on the recognised impact of contour interaction on amblyopia sensitivity with other tests. Additional studies comparing the sensitivity of the Lea tests to line and surrounded letter acuity in amblyopic patients, such as has been done for the Glasgow test, ${ }^{10}$ would be helpful.

How should we currently be testing preschool visual acuity? It would seem that the best visual acuity measurement strategy for children over age 4 years would be to use a single surrounded letter optotype in a logMAR progression ${ }^{18}$ or the Glasgow test..$^{8-10}$ For the developmentally delayed and children unable to perform these letter tests, the use of the surrounded Lea symbols test is a reasonable alternative. ${ }^{18}$ Another option would be the " $\mathrm{O}$ " test, which had a testability of $90 \%$ in a group of 62 mildly delayed 4-10 year old children. ${ }^{19}{ }^{20}$ Practitioners should have more than one test available, as not one of these tests can be used successfully in all young children. However, many young children will not be testable with any of the currently available tests.

Picture based test optotypes are clearly necessary to quantitatively measure the visual acuity of young children. Clinicians must continue to refine as well as develop new visual acuity tests for preschool children, which combine picture optotypes, which have high testability, with crowding features ensuring sufficient sensitivity to detect amblyopia.

Br J Ophthalmol 2002;86:489-490

\section{Author's affiliations}

M X Repka, Johns Hopkins University School of Medicine, 233 Wilmer, Johns Hopkins Hospital, 600 North Wolfe Street, Baltimore, MD 21287-9028, USA

\section{REFERENCES}

1 Bailey IL, Lovie JE. New design principles for visual acuity test charts. Am J Optom Physiol Opt 1976:53:745-53.

2 Ferris FL, Kassoff A, Bresnick GH, et al. New visual acuity charts for clinical research. Am J Ophthalmol 1982;94:91-6.

3 Jenkins PF, Prager TC, Mazow ML, et al. Preliterate vision screening: a comparative study. Am Orthopt J 1983;33:91-8.

4 Mayer DL, Gross RD. Modified Allen pictures to assess amblyopia in young children. Ophthalmology 1990;97:827-32.

5 Simons K. Visual acuity norms in young children. Surv Ophthalmol 1983;28:84-92.
6 Flom MC, Heath GG, Takahashi E. Contour interaction and visual resolution: contralateral interaction and visual resolution: contral
effects. Science 1963;142:979-80.

7 Stager DR, Everett ME, Birch EE. Comparison of crowding bar and linear optotype acuity in amblyopia. Am Orthoptic J 1990:40:51-6.

8 Holmes JM, Beck RW, Repka MX, et al. The amblyopia treatment study visual acuity testing protocol. Arch Ophthalmol

2001;1 19:1345-53

9 McGraw PV, Winn B. Glasgow acuity cards: a new test for the measurement of letter acuity in children. Ophthalmic Physiol Opt 1993:13:400-4.

10 McGraw PV, Winn B, Gray LS, et al. Improving the reliability of visual acuity measures in young children. Ophthalmic Physiol Opt 2000;20:173-84.

11 Simmers AJ, Gray LS, Spowart K. Screening for amblyopia: a comparison of paediatric letter tests. Br J Ophthalmol 1997;81:465-9.

12 Fern KD, Manny RE. Visual acuity of the preschool child: a review. Am J Optom Physiol Opt 1986;63:319-45.

13 Hyvärinen L, Nasanen R, Laurinen P. New visual acuity test for pre-school children. Acta Ophthalmol 1980;58:507-11

14 Hered R, Murphy S, Clancy M. Comparsion of the HOTV and Lea symbols charts for preschool vision screening. J Pediatr Ophthalmol Strabismus 1997:34:24.

15 Becker R, Hubsch S, Graf MH, et al. Premlinary report: examination of young children with Lea symbols. Strabismus 2000;8:209-13

16 Miller JM, Dobson V, Harvey EM, et al. Comparison of preschool vision screening methods in a population with a high prevalence of astigmatism. Invest Ophthalmol prevalence of astigmatism.
Vis Sci 2001;42:917-24.

17 Graf M, Becker R, Kaufmann H. Lea symbols: visual acuity assessment and detection of amblyopia. Graefes Arch Clin Exp Ophthalmol 2000;238:53-8.

18 Hartmann E, Dobson V, Hailine L, et al. Preschool vision screening: summary of a task force report. Pediatrics 2000;106:1105.

19 Lithander J. Two techniques to evaluate visual acuity from the age of 18 months. Strabismus 1996;4:15-23.

20 Lithander J. Visual development in the healthy eye from 24 months to four years of age. Acta Ophthalmol 1997;75:275-6.

\section{Gene therapy: new "magic bullets" to prevent ocular scarring}

\section{P T Khaw, A D Cambrey, G A Limb, J T Daniels}

\section{Will the advances in modern molecular biology open doors to new therapies?}

$T^{3}$ he processes involved in ocular scarring play a part in either the pathogenesis or failure of treatment of most of the major blinding diseases in the world. These processes include capsular opacification and contraction after cataract surgery. Although posterior capsular opacification is relatively easily treated with laser, this biological process poses great problems following cataract surgery in developing countries, and will inhibit the development of a true accommodating lens replacement. The retinal scarring that occurs in proliferative vitreoretinopathy (PVR) and macular degeneration is also an important example of the blinding scarring process.

The scarring process following glaucoma filtration surgery is one of the best examples of the importance of being able to control healing in virtually all patients having a particular procedure. Recent data from the NIH advanced glaucoma intervention study (AGIS) ${ }^{1}$ have shown that individuals with the lowest intraocular pressures (average $12.3 \mathrm{~mm}$ $\mathrm{Hg}$ ) had virtually no overall glaucomatous progression over nearly a decade. The healing response after surgery is the main long term determinant of long term intraocular pressure. Therefore, if we are able to control the healing response in all patients after glaucoma surgery, it offers us the tantalising prospect of minimal or no disease progression in the vast majority of our glaucoma patients, even those with advanced disease.

The advent of anticancer agents has revolutionised glaucoma surgery in patients who have a high risk of failure following surgery. Their use has now been extended to patients with a lower risk of surgical failure in an attempt to achieve lower final intraocular pressures. However, these agents are relatively nonspecific and exert their action by causing cellular growth arrest and widespread cell death. It is therefore still difficult to 
titrate the effects, and many side effects occur. These include corneal toxicity associated with 5-fluorouracil injections because this drug is non-specific and kills epithelial cells as well. Other side effects include hypotony, and thin cystic drainage blebs that are associated with blinding side effects such as hypotony and endophthalmitis. Therefore the search continues for novel treatments that can control the wound healing response without the side effect profile seen with current anticancer agents.

Will the advances in modern molecular biology open doors to new therapies? The molecular knowledge of enzyme systems in non-mammalian systems allows us to transfect human cells with these enzymes. This allows us to specifically target only cells that have been transfected with these genes. Drugs that require these enzymes to be activated target the transfected cells-hence the term "magic bullet."

\section{if we are able to control the healing response in all patients after glaucoma surgery, it offers us the tantalising prospect of minimal or no disease progression in the vast majority}

In this issue of the BJO (p 581), Akimoto et al report an innovative combination of existing anticancer agents and the new gene therapies. Tenon's fibroblasts were transfected with an enzyme called cytosine deaminase, which is only found in bacteria and fungi. 5-Fluorocytosine is a non-toxic prodrug that is converted to 5 -fluorouracil by cytosine deaminase. This gene was then inserted into genetically marked Tenon's fibroblasts, which were implanted subconjunctivally. Cell death appeared to occur only in cells transfected with the enzyme gene, without any corneal toxicity. In theory, if cells in a trabeculectomy area could be transfected with this gene, then topical drops could be applied affecting only the cells in the wound area. This moves us closer to the magic bullet so coveted in cancer chemotherapy.

There are still many hurdles to overcome. Adenovirus transfer to the subconjunctival space can be achieved successfully, but transfection is only transient with a peak at 7 days and elimination by 14 days. ${ }^{2}$ However, inhibition of scarring in the early period of scarring may be all that is necessary in most patients. After all, this is part of the basis of single intraoperative applications of anticancer agents. ${ }^{3}$ Longer lasting treatments may be required for more aggressive prolonged scarring which occurs in higher risk patients.

Adenovirus vectors do have disadvantages that include a host immune reaction which increases with repeated use, and non-specific transfection of all cells. Modifications of the adenovirus vector may overcome many of these problems. ${ }^{4}$ Other virus vectors may have advantages, ${ }^{5}$ but the fact that they integrate with the host genome may make them less desirable, particularly in less permanent situations such as scarring after surgery. ${ }^{6}$ Advances in the type of vectors will be very important in determining how much this type of therapy becomes clinical reality in the near future. ${ }^{7}$

Advances in our understanding of molecular biology also offer us other exciting forms of gene therapy. Certain growth factors found in damaged tissues stimulate healing and scarring. One growth factor, transforming growth factor $\beta$ (TGF- $\beta$ ), stimulates more ocular fibroblast scarring activity than other growth factors. ${ }^{8}$ Fetal wound healing, which is associated with scarless healing, has an environment that is lacking in TGF- $\beta$. The local production of these growth factors can be inhibited by blocking or destroying the RNA molecules that encode the production of this growth factor. This achieves highly specific control of one arm of wound healing leaving others intact. This can be done using short protected chains of DNA (antisense oligonucleotides) or by inserting the genes encoding for enzymes called ribozymes which destroy precise sequences of RNA. ${ }^{9}$ Advanced molecular techniques also permit the creation of another type of "magic bullet" by facilitating the selection of immunoglobulin genes and the synthesis of highly specific human antibodies to TGF- $\beta 2$. These antibodies have been shown to be effective in preventing scarring in a model of filtration surgery ${ }^{10}$ and resulted in reduced final intraocular pressures in a pilot human study without the thin cystic blebs seen in eyes treated with anticancer agents. ${ }^{11}$ Future advances in our understanding of genotype, perhaps helped by gene microarrays, may help us to identify groups of patients that scar more aggressively and also identify subgroups that may respond better to certain treatments.

Finally, it is appropriate that the current paper by Akinoto et al combines both old and new technologies. In our rush to embrace modern molecular medicine, we must not forget that existing treatments may still have much to offer. A simple change in the technique of antimetabolite application has reduced our long term bleb related complications in a high risk group from 15\% to $0 \%{ }^{12}$ Based on simple cell culture modelling, ${ }^{13}$ the use of a inexpensive continuous infusion of 5-fluorouracil combined with heparin has more than halved the incidence of PVR in a high risk group from $26 \%$ to $11 \%,{ }^{14}$ the first randomised clinical trial to show that PVR could be significantly reduced. It is likely that there are many more "hidden treasures" in combinations of existing and new treatments, an analogy being the $90+\%$ "cure" rates seen in some previously untreatable cancers with combinations of old and new treatments. There are literally millions of patients undergoing surgical treatments that could benefit if these treasure chests can be unlocked. The keys lie in the commitment and support for future basic and clinical research.

\section{ACKNOWLEDGEMENTS}

The authors are supported by the Guide Dogs for the Blind, RNIB, Wellcome Trust 062290, and the Medical Research Council (UK) grant G9330070.

Br J Ophthalmol 2002;86:490-492

\section{Authors' affiliations}

P T Khaw, A D Cambrey, G A Limb, J T Daniels, Wound Healing Research Unit, Epithelial Repair and Regeneration Group, Divisions of Pathology, Cell Biology and Glaucoma, Moorfields Eye Hospital and the Institute of Ophthalmology, University College London, UK

Correspondence to: P T Khaw, Wound Healing Research Unit, Glaucoma Unit and Divisions of Pathology and Cell Biology, Moorfields Eye Hospital and Institute of Ophthalmology, Bath Street, London ECIV 9EL, UK.

p.khaw@ucl.ac.uk

\section{REFERENCES}

1 AGIS Investigators. The Advanced Glaucoma Intervention Study (AGIS) 7. The relationship between control of intraocular pressure and visual field deterioration. $\mathrm{Am} J$ Ophthalmol 2000;130:429-40.

2 Skaf M di Martino DS, de Arruda Mello PA et al. Adenoviral-mediated gene transfer to the filtering bleb in rabbits. J Glaucoma $2001 ; 10: 470-6$

3 Khaw PT, Sherwood MB, MacKay SLD, et al. 5-Minute treatments with fluorouracil, floxuridine and mitomycin have long-term effects on human Tenon's capsule fibroblasts. Arch Ophthalmol 1992;110:1150-4.

4 Thomas CE, Schiedner G, Kochanek S, et al. Peripheral infection with adenovirus causes unexpected long-term brain inflammation in animals injected intracranially with first-generation, but not with high-capacity, adenovirus vectors: toward realistic long-term neurological gene therapy for chronic diseases. Proc Natl Acad Sci USA diseases. Proc Natl
2000;97:7482-7.

5 Trono $D$. Lentiviral vectors: turning a deadly foe into a therapeutic agent. Gene Ther 2000;7:20-3.

6 Hollingsworth SJ, Barker SG. Gene therapy: into the future of surgery. Lancet 1999;353:S1 19-20.

7 Anderson WF. Human gene therapy. Nature 1998:392:25-30.

8 Khaw PT, Occleston NL, Schultz GS, et al. Activation and suppression of fibroblast activity. Eye 1994;8:188-95.

9 Lewin AS, Drenser KA, Hauswirth WW, et al. Ribozyme rescue of photoreceptor cells in a transgenic rat model of autosomal dominant 
retinitis pigmentosa. Nat Med 1998;4:967-71.

10 Cordeiro MF, Gay JA, Khaw PT. Human anti-transforming growth factor-beta2 antibody: a new glaucoma anti-scarring agent. Invest Ophthalmol Vis Sci 1999;40:2225-34.

11 Siriwardena D, Khaw PT, King AJ, et al. A randomised double-masked placebo-contolled trial of human anti-TGFbeta2 monoclonal antibody (CAT-152): a potential new modulator of wound healing following trabeculectomy. Ophthalmology 2002;109:427-81.

12 Wells AP, Cordeiro MF, Bunce CV, et al. Cystic bleb related complications in limbus versus fornix based flaps in paediatric and young adult trabeculectomy with high dose mitomycin C. Invest Ophthalmol Vis Sci 2001;42:S544.
13 Kon CH, Occleston NL, Foss A, et al. Effects of single, short-term exposures of human retinal pigment epithelial cells to thiotepa or 5-fluorouracil: implications for the treatment of proliferative vitreoretinopathy. $\mathrm{Br}$

Ophthalmol 1998;82:554-60.

4 Asaria $\mathrm{RH}$, Kon $\mathrm{CH}$, Barin prevents 5 -fluorouracil and heparin prevents proliferative vitreoretinopathy: results from randomised double trial. Ophthalmology 2001;108:1179-83.

\section{Want full access but don't have a subscription?}

\section{Pay per access}

For just US $\$ 25$ you can have instant access to the whole website for 30 days. During this time you will be able to access the full text for all issues (including supplements) available. You will also be able to download and print any relevant pdf files for personal use, and take advantage of all the special features British Journal of Ophthalmology online has to offer.

www.bjophthalmol.com 